Sample size and the stability of correlation coefficients: A replication of Schönbrodt \& Perugini (2013)

Gabriela Hofer ${ }^{1}$

${ }^{1}$ University of Graz, Institute of Psychology

Author Note

This report was prepared as coursework for a doctoral course on "R-based simulation techniques, Monte Carlo and bootstrapping for the investigation of psychological issues" taught by Dr. Guilherme Maia de Oliveira Wood at the University of Graz in the spring term 2020. The underlying project will likely undergo further development and this manuscript will be updated accordingly.

Correspondence concerning this article should be addressed to Gabriela Hofer, University of Graz, Institute of Psychology, Universitätplatz 2, 8010 Graz, Austria. E-mail: gabriela.hofer@uni-graz.at 


\begin{abstract}
The sample size in a quantitative study does not only affect statistical power but also the precision with which effects can be estimated. Sch?nbrodt and Perugini (2013) have applied Monte-Carlo simulations to establish the point of stability of correlation coefficients (i.e., the sample size from which on they only fluctuate around the true value within acceptable margins). They reported that the sample size necessary to achieve stability depends on the size of the underlying correlation, the width of the margins within which fluctuations are tolerated (the corridor of stability), and the desired confidence that the correlation does not exceed these margins. According to their suggestion, a sample around 250 is desirable for typical scenarios in personality psychology. The present contribution aimed to replicate these findings and extend them by determining the point of stability for $\rho=.0$ and very narrow corridors of stability. Results pertaining to the replication were virtually identical to those reported by the original authors. In addition, correlations of .0 to became stable within a corridor of .1 at around 260 participants. Considerably more data points should be collected if only correlations within a very narrow corridor of stability are to be accepted. Future research could extend these findings by investigating the point of stability in data with different types of non-normal distributions.
\end{abstract}

Keywords: sample size planning, point of stability

Word count: 2493 
Sample size and the stability of correlation coefficients: A replication of Schönbrodt \& Perugini (2013)

In recent years, it has become apparent that many psychological findings are less robust than one might have expected (e.g., Open Science Collaboration, 2015). These insights have led to what some researchers call the credibility revolution (Vazire, 2018), a period marked by increased self-scrutiny within psychological research and calls for improvements targeting different parts of the research cycle (see e.g., Funder et al., 2014; Munafò et al., 2017; Simmons, Nelson, \& Simonsohn, 2011). Sample size planning is one area that is often included in suggestions for increased robustness and is at the center of the present report.

Within the null hypothesis significance testing framework, the sample size to be collected in a study should ideally not only depend on the availability of time and other resources, but also on careful power analyses (Cohen, 1992). To put it very simply, we want to ensure that the amount of data we collect is sufficient to find (i.e., to mark as significant) the effect that we are interested in (if it actually exists in the population). However, many researchers have argued that it might be worthwhile to not only look at the significance of effects but also at the degree of certainty or uncertainty around them, particularly when it comes to investigations of replicability (for a summary of arguments see e.g., Lakens \& Evers, 2014). Consider a correlation of $r=.5$ that we might find in small sample of $n=20$ or a larger sample of $n=200$. While this correlation is significant at $p<.05$ in both samples, the associated confidence interval at $n=20[95 \%$ CI .074; .772] encloses both effects that are close to zero and large effects, whereas the one at $n=200$ [95\% CI .388; .597] narrows down around a medium to large effect. Thus, while findings from both samples speak for a population effect that is different from zero, those from the smaller sample give us only little certainty about the potential size of this effect.

A question related to sample size considerations and the uncertainty about the size of 
correlations is at which sample size correlations stabilize. It has been noted that the magnitude of correlations fluctuates widely in small samples (for a graphical illustration see Schönbrodt \& Perugini, 2013). As a basic requirement for robustness, however, we might want to collect enough data that any further addition or removal of single data points does not lead to drastic changes in our main results. In a well-received simulation study, Schönbrodt and Perugini (2013) have looked at the number of participants that is necessary for a correlation in a sample to stabilize around the population correlation. More specifically, they have investigated at which sample size a correlation coefficient of a given size no longer leaves a desired corridorofstability $(C O S)$ of a width $w^{1}$ around the population correlation $\rho$. The outcome the authors were interested was the pointof stability $(P O S)$; that is the sample size from which on the correlations no longer leave the desired $C O S$.

Schönbrodt and Perugini simulated normally distributed data with population correlations ranging from $\rho=.1$ to $\rho=.7$ and drew 100,000 samples with $n=1,000$ from this data. They then computed the correlations for subsamples of increasing size, starting from $n_{\min }=20$ and ranging to $n_{\max }=1,000$ with a step size of 1 . Finally, they calculated the POS from this data for $C O S$ with three different widths $(w=.10, .15$, and .20$)$ and 3 different levels of confidence $(80 \%, 90 \%$, and $95 \%)$. Their results indicated that the point of stability depends on all three factors they varied. The number of participants necessary to obtain stable correlation coefficients was higher for (1) low population correlations, (2)

${ }^{1}$ Schönbrodt and Perugini (2013) had originally specified the width $w$ of the COS in units of $q$, an effect size metric that - due to its Fisher- $r$-to- $Z$-transformation — is not affected by the magnitude of the correlation but only by the sample size. Thus, the $C O S$ with a width of $w \pm .10$ around $\rho=.30(.31$ after the Fisher-r-to- $Z$-transformation) would be $C O S_{.10}=[.21 ; .39]$. In a corrigendum, Schönbrodt and Perugini (2018) noted that their results were actually based on non-transformed corridors around the population correlation (e.g., the $C O S .10$ for a $\rho=.3$ was $[.2 ; .4]$ ) due to an error in their code. They argued that, while this approach did not match the one they had described in their paper, it might actually be better than the one including the Fisher- $r$-to- $Z$-transformation because its results are easier to interpret. 
small corridors of stability, and (3) higher desired confidence. According to the authors, for the rather small effects of $r$ around .2 that are common in personality psychology (Richard, Bond, \& Stokes-Zoota, 2003; see also Gignac \& Szodorai, 2016), sample size should be at least $n=238$, if researchers want to remain within a small $\operatorname{COS}(w=.10)$ with a confidence level of $80 \%$. The authors also imposed the different population correlations on real-world data with slight deviations from normality that are typical to psychological data. Here, they found that correlations stabilized only slightly later than with normally distributed data.

\section{The present simulation study}

The aim of the present simulation study was to replicate and extend the one conducted by Schönbrodt and Perugini (2013). The following extensions were made to the original study: (1) Recently, it has often been argued that there are many cases in which the absence an of effect might be of considerable scientific interest and practical importance (e.g., Harms \& Lakens, 2018). One goal of the present study was, therefore, to determine at which sample size researchers can be confident that population correlations of 0 remain within an acceptable corridor of stability. (2) Particularly when researchers are interested in null-effects or small effects, they might only want to tolerate very small fluctuations around the population correlation. While this might not be feasible for many research projects (Schönbrodt \& Perugini, 2013), studies stemming from big collaborative projects like the Psychological Science Accelerator (Moshontz et al., 2018) might benefit from information on the sample size that is required for a narrow COS. For this reason, I included a smaller COS of $w=.05$ in my analyses. (3) Although initially planned otherwise (see Footnote 1), Schönbrodt and Perugini (2013) conducted their simulations with $w$ s of the COS in the $r$ metric. However, since their original argument in favor for using a COS that has the same meaning for different population correlations - i.e., one in the Fisher-Z-metric - appears well-founded, results of the present simulation will be 
reported with and without the transformation.

\section{Methods}

The Monte-Carlo simulations reported here have been conducted in $R$ (R Core Team, 2020) using the MASS package (Ripley et al., 2020) for simulating multivariate normal data, the tidyverse collection (Wickham \& RStudio, 2019) for data wrangling, the psych package (Revelle, 2020) for Fisher- $r$-to- $Z$-transformations, the doParallel package (Ooi, Corporation, Weston, \& Tenenbaum, 2019) for parallel computations, and the papaja package (Aust \& Barth, 2020) for scientific reporting. In many instances, the source code (see https://osf.io/ydbwr/) provided by Schönbrodt and Perugini $(2013,2018)$ was a valuable starting point. The complete source code for this simulation can be found on https://osf.io/fyuz4/. In the following, all steps of the simulation including all major deviations from the original study by Schönbrodt and Perugini (2013) will be described:

1. Simulate a "population" - that is a bivariate Gaussian distribution with $1,000,000$ cases - for each of 8 prespecified correlations ( $\rho$ ranging from .0 , .to .7 as compared to $\rho$ ranging from .1 to .7 in the original study). To this end, I used the mvrnorm function of the MASS package instead the approach applied by the original authors (described by Ruscio \& Kaczetow, 2008).

2. Draw 100,000 samples with $n=1,000$ from each of these populations.

3. For each of these samples, calculate the correlation for the first $n_{\min }=20$ to $n_{\max }=$ 1,000 observations with a step size of 1 .

4. Specify the different widths of the COS that the sample correlations of the different trajectories should be compared to $(w=.05, .10, .15$, and .20 where $w=.05$ was an addition to the original study). As already stated, in the present study analyses were conducted both with Fisher-r-to- $Z$-transformed $w$ s and $w$ s in the $r$-metric. 
5. Compute the POS for each bootstrap sample by comparing each $r$ - starting from the one at $n_{\max }=1,000$ - with the bounds of the respective $C O S$ in order to determine the lowest sample size for which the $r$ still remains within the bounds.

6. Compute the $80 \%, 90 \%$, and $95 \%$ percentiles of $P O S$ values for each $C O S$ and $\rho$.

\section{Results and Discussion}

Table 1 shows the main results of this simulation: Its upper part contains the $P O S_{\text {crit }}$ for different combinations of $\rho \mathrm{s}, w \mathrm{~s}$ (in the $r$ metric like in the original simulation), and levels of confidence; in its lower part the $P O S_{\text {crit }}$ based on different $w$ s in the $Z$ metric can be found. As Schönbrodt and Perugini (2018) already noted, findings based on the $Z$ metric are not expected to vary between $\rho$ s, since the underlying effect size measure does not depend on the strength of the correlation. This was also the case in this simulation, apart from some minor fluctuations $\left(n_{\text {maxdiff }} \pm 5\right)$, for which the highest respective $P O S_{\text {crit }}$ was stored in Table 1.

\section{Replication}

When comparing the results for $w$ s between .10 and .20 and $\rho$ s between .1 and .7 from Table 1 to those reported by Schönbrodt and Perugini (2013), it becomes apparent that they are virtually identical. As an example, where in the original simulation the $P O S_{\text {crit }}$ for a small effect of $\rho=.1$ and a small corridor of stability with $w=.10$ was at 252 participants, it lay at 255 participants in the present sample. Like in the original study, higher levels of desired confidence, lower widths of the corridor of stability, and lower population correlations were associated with later critical points of stability (i.e., higher sample size demands).

While the source code provided by Schönbrodt and Perugini was an important starting point for this project, quite some alterations have been made to it. The robustness 
of the findings despite these changes is encouraging. Additionally, the code used in the original simulation did not contain a seed and results would not have been completely reproducible, even when executing the original code. The slight deviations between the results of this and the original simulation are, therefore, negligible.

\section{Extension}

In addition to the replication of the simulations reported by Schönbrodt and Perugini (2013), the present study aimed to investigate the $P O S_{\text {crit }}$ for $\rho$ s of zero and corridors of stability with a very narrow width $(w=.05)$, as well as, to provide the $P O S_{\text {crit }}$ based $w$ s in the $Z$ metric (as was originally intended by Schönbrodt \& Perugini).

When observing the results for $\rho=.0$ in the first row of Table 1 , it is noticeable how similar they are to those for small correlations. In scenarios where one wants sample correlations to remain within .1 of a population correlation of zero - and thus just below what most would consider a small effect — with a confidence level of 80\%, data from 258 cases should be collected.

As can be seen in the last row of Table 1, results for $w$ s in the $Z$ metric were comparable to those of $w$ s in the $r$ metric for $\rho$ s of zero. This is in line with the fact, that at low correlation sizes, Fisher's $Z$ corresponds closely to $r$ - with a correlation of zero also being zero in the $Z$ metric. Thus, researchers deciding to base their sample size planning on a corridor of stability that corresponds to a small effect $(w=.1)$ and is comparable across correlations of different sizes, would — again — be advised to collect a sample of around 260 to obtain a confidence level of $80 \%$ (see also Schönbrodt \& Perugini, 2018) and about 120 participants, if slightly larger deviations from the population correlation are to be tolerated $(w=.15)$.

If researchers are only willing to tolerate very low fluctuations around the population correlation $(w=.05)$, this leads to considerably higher sample size demands. In the $Z$ 
metric or for $\rho=.0$ in the $r$ metric and with a desired level of confidence of $80 \%$, sample size should be around 730 participants; for $\rho=.2$ it should still be close to 710 . For very high correlations of $\rho=.7$, which one might, for example, encounter in studies dedicated to questionnaire construction and validity, this level of stability can already be reached with 271 participants. However, results for $w=.05$ - particularly those for small $\rho$ s — should only be interpreted with caution, since a considerable number of the respective bootstrap samples did not yield a point of stability within $n_{\max }=1,000$ (see Table 2). Over all ws, for $98.34 \%$ of all samples the POS could be determined within $n_{\max }=1,000$, when $w$ was defined in units of $r$; when $w$ was defined in units of $Z, 97.07 \%$ of samples stabilized within the maximal sample size. Thus, a sample size of more than 1,000 might be needed for more reliable results about the $P O S_{\text {crit }}$ for very small $w$ s around small $\rho$ s and the present results are likely an underestimation of the true sample size needed for stabilization.

\section{Conclusion and future research}

This simulation project replicated the findings on the point of stability of correlation coefficients that were reported by Schönbrodt and Perugini (2013). The conclusion that "in typical scenarios the sample size should approach 250 for stable estimates" (p. 609) remains valid and can also be extended to correlations of zero. Initial evidence indicates that the sample size needed for a very narrow corridor of stability is considerably larger but might not be out of reach for large-scale collaborations. These findings already provide researchers that are planning their studies with important information. Nevertheless, there are still many interesting questions about the stabilization of correlations that remain to be answered. As the original authors have already argued, the perfectly normally distributed data their main simulation — and, by extension, also the present one - is based on, are far from the real-life data obtained in psychological studies. The authors have also explored the POS in some real-life datasets, on which they had imposed their prespecified population correlations, and have found that (slightly) non-normal data has little impact 
on the POS. However, a more systematic investigation of the effects of different levels skewness and kurtosis on the POS might provide researchers with the possibility to make even more informed decisions when it comes to sample size planning. 


\section{References}

Aust, F., \& Barth, M. (2020). papaja: Create APA manuscripts with $R$ Markdown. Retrieved from https://github.com/crsh/papaja

Cohen, J. (1992). A power primer. Psychological Bulletin, 112(1), 155-159. https://doi.org/10.1037/0033-2909.112.1.155

Funder, D. C., Levine, J. M., Mackie, D. M., Morf, C. C., Sansone, C., Vazire, S., \& West, S. G. (2014). Improving the dependability of research in personality and social psychology: Recommendations for research and educational practice. Personality and Social Psychology Review, 18(1), 3-12.

https://doi.org/10.1177/1088868313507536

Gignac, G. E., \& Szodorai, E. T. (2016). Effect size guidelines for individual differences researchers. Personality and Individual Differences, 102, 74-78. https://doi.org/10.1016/j.paid.2016.06.069

Harms, C., \& Lakens, D. (2018). Making 'null effects' informative: Statistical techniques and inferential frameworks. Journal of Clinical and Translational Research, 3(Suppl 2), 382-393. Retrieved from https://www.ncbi.nlm.nih.gov/pmc/articles/PMC6412612/

Lakens, D., \& Evers, E. R. K. (2014). Sailing from the seas of chaos into the corridor of stability: Practical recommendations to increase the informational value of studies. Perspectives on Psychological Science, 9(3), 278-292. https://doi.org/10.1177/1745691614528520

Moshontz, H., Campbell, L., Ebersole, C. R., IJzerman, H., Urry, H. L., Forscher, P. S., ... Chartier, C. R. (2018). The Psychological Science Accelerator:

Advancing psychology through a distributed collaborative network. Advances in Methods and Practices in Psychological Science, 1(4), 501-515. 
https://doi.org/10.1177/2515245918797607

Munafò, M. R., Nosek, B. A., Bishop, D. V. M., Button, K. S., Chambers, C. D., Percie du Sert, N., ... Ioannidis, J. P. A. (2017). A manifesto for reproducible science. Nature Human Behaviour, 1(1), 0021. https://doi.org/10.1038/s41562-016-0021

Ooi, H., Corporation, M., Weston, S., \& Tenenbaum, D. (2019). doParallel: Foreach Parallel Adaptor for the 'parallel' Package. Retrieved from https://CRAN.R-project.org/package=doParallel

Open Science Collaboration. (2015). Estimating the reproducibility of psychological science. Science, 349 (6251), aac4716. https://doi.org/10.1126/science.aac4716

R Core Team. (2020). R: A language and environment for statistical computing. Vienna, Austria: R Foundation for Statistical Computing. Retrieved from https://www.R-project.org/

Revelle, W. (2020). Psych: Procedures for Psychological, Psychometric, and Personality Research. Retrieved from https://CRAN.R-project.org/package=psych

Richard, F. D., Bond, C. F., \& Stokes-Zoota, J. J. (2003). One hundred years of social psychology quantitatively described. Review of General Psychology, 7(4), 331-363. https://doi.org/10.1037/1089-2680.7.4.331

Ripley, B., Venables, B., Bates, D. M., 1998), K. H. (. port ca, 1998), A. G. (. port ca, \& Firth, D. (2020). MASS: Support Functions and Datasets for Venables and Ripley's MASS. Retrieved from https://CRAN.R-project.org/package=MASS

Ruscio, J., \& Kaczetow, W. (2008). Simulating multivariate nonnormal data using an iterative algorithm. Multivariate Behavioral Research, 43(3), 355-381. https://doi.org/10.1080/00273170802285693 
Schönbrodt, F. D., \& Perugini, M. (2013). At what sample size do correlations stabilize? Journal of Research in Personality, 47(5), 609-612. https://doi.org/10.1016/j.jrp.2013.05.009

Schönbrodt, F. D., \& Perugini, M. (2018). "At what sample size do correlations stabilize?": Corrigendum. Journal of Research in Personality, 74, 194-194. https://doi.org/10.1016/j.jrp.2018.02.010

Simmons, J. P., Nelson, L. D., \& Simonsohn, U. (2011). False-positive psychology: Undisclosed flexibility in data collection and analysis allows presenting anything as significant. Psychological Science, 22(11), 1359-1366. https://doi.org/10/bxbw3c

Vazire, S. (2018). Implications of the credibility revolution for productivity, creativity, and progress. Perspectives on Psychological Science, 13(4), 411-417. https://doi.org/10.1177/1745691617751884

Wickham, H., \& RStudio. (2019). Tidyverse: Easily Install and Load the 'Tidyverse'. Retrieved from https://CRAN.R-project.org/package=tidyverse 


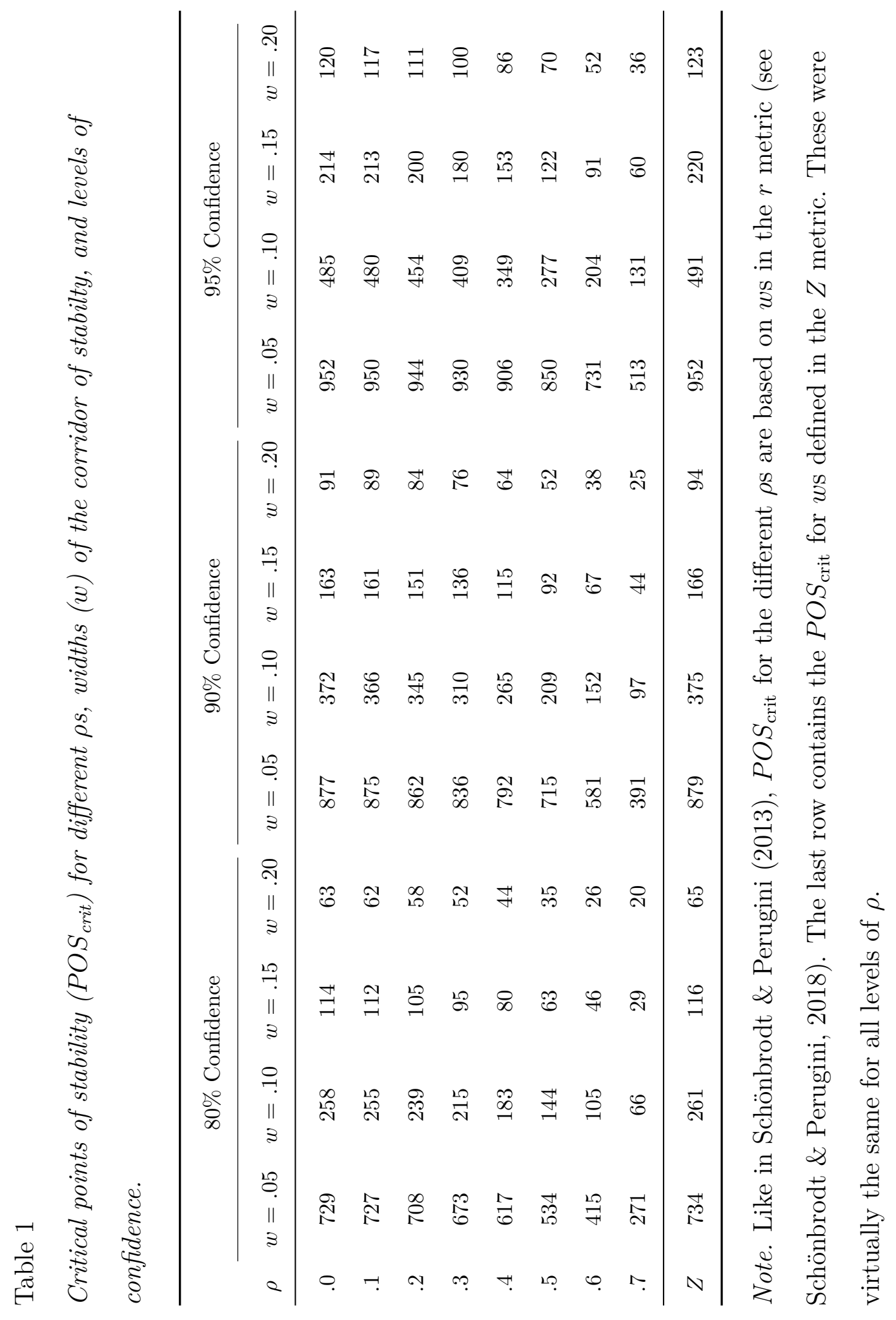


Table 2

Percentage of bootstrap samples

containing a POS at $n \leq 1,000$.

\begin{tabular}{ccccc}
\hline & \multicolumn{5}{c}{$w$} \\
\cline { 2 - 5 }$\rho$ & .05 & .10 & .15 & .20 \\
\hline .0 & 88.48 & 99.85 & 100.00 & 100.00 \\
.1 & 88.82 & 99.86 & 100.00 & 100.00 \\
.2 & 89.89 & 99.90 & 100.00 & 100.00 \\
.3 & 91.60 & 99.95 & 100.00 & 100.00 \\
.4 & 93.88 & 99.98 & 100.00 & 100.00 \\
.5 & 96.36 & 99.99 & 100.00 & 100.00 \\
.6 & 98.54 & 100.00 & 100.00 & 100.00 \\
.7 & 99.78 & 100.00 & 100.00 & 100.00 \\
\hline$Z$ & 88.44 & 99.83 & 100.00 & 100.00
\end{tabular}

Note. The last row contains the

respective percentages for $w$ s defined

in the $Z$ metric, which were the same

for all levels of $\rho$. All other rows

contain percentages for $w$ s in the $r$

metric. 